\title{
Alcuni spunti di riflessione offerti dalla lettura della Costituzione dello stato di Aguascalientes
}

\author{
Paolo PASSAGLIA*
}

\begin{abstract}
Sommario: I. Premessa. II. Un stato piccolo, ma con una storia importante. III. La "continuità nel cambiamento". IV. La "manutenzione costituzionale". V. Il paradosso delle riforme. VI. Una Costituzione "ricca".
\end{abstract}

\section{PREMESSA}

L'esame del testo costituzionale dello stato di Aguascalientes si rivela di grande interesse per il giurista straniero, anche per gli spunti che offre nella prospettiva di una analisi di diritto comparato.

Con riferimento a taluni aspetti, questo interesse è particolarmente forte per un giurista italiano, perché la Costituzione di Aguascalientes consente di porsi domande anche su alcuni elementi che sono propri dell'evoluzione costituzionale conosciuta dall'Italia negli ultimi lustri. Su tali caratteri ci si soffermerà principalmente, anche perché la ricchezza della Costituzione impedisce di fare una esposizione che possa dirsi anche solo blandamente compiuta dei suoi contenuti. D'altra parte, la conoscenza molto limitata che chi scrive ha dell'ordinamento non consentirebbe in alcun modo di porsi sul piano dell'analisi concreta degli istituti giuridici.

* Professore ordinario di Diritto comparato, Università di Pisa, Italia. Coordinatore scientifico pro tempore dell'Area di Diritto Comparato del Servizio Studi, Corte Costituzionale della Repubblica Italiana. Contacto: paolo.passaglia@unipi.it; https://orcid. org/0000-0002-5298-2996. 
Ci si limiterà, allora, a comunicare alcune «sensazioni» (poiché di sensazioni si tratta, più che di una analisi vera e propria), maturate nel corso della lettura della Costituzione di Aguascalientes e dello studio volto alla sua contestualizzazione storico-istituzionale.

\section{UN STATO PICCOLO, MA CON UNA STORIA IMPORTANTE}

Lo stato di Aguascalientes è tra i più piccoli e tra i meno popolosi stati della Federazione messicana. Questa constatazione non può in alcun modo condurre a ritenere marginale l'evoluzione costituzionale che ha caratterizzato questo territorio. Il riferimento - fin troppo scontato- è all'importanza, per la storia costituzionale del Messico, avuta dalla Convención de Aguascalientes, che si celebrò tra il 10 ottobre ed il 9 novembre 1914, e che segnò una tappa fondamentale nell'edificazione del nuovo ordine successivo alla rivoluzione. Lo studio della storia istituzionale dello stato di Aguascalientes, peraltro, suggerisce molto di più del semplice richiamo a quel frangente storico, per quanto importante esso sia: il costituzionalismo locale, a far tempo dal 1857, quando lo stato fu istituito, ${ }^{1}$ è, infatti, assai ricco di argomenti ed implicazioni. Ad alcuni di questi si farà accenno nelle pagine che seguono.

\section{LA “CONTINUITÀ NEL CAMBIAMENTO”}

Un primo elemento che merita essere segnalato attiene all'esistenza di una sorta di "continuità nel cambiamento" che sembra caratterizzare la storia costituzionale dello stato di Aguascalientes.

Sin dalla creazione di un ente istituzionalmente autonomo, Aguascalientes ha mostrato un marcato dinamismo costituzionale, che si è tradotto nell'elaborazione di quattro carte costituzionali e di un numero estrema-

1 Sarebbe forse più corretto, peraltro, indicare come dies a quo il 1835, anno in cui il territorio di Aguascalientes venne separato da Zacatecas per diventare un territorio autonomo. Sulla creazione dello stato di Aguascalientes, González Oropeza, Manuel e Cienfuegos Salgado, David, Digesto Constitucional Mexicano. Aguascalientes, México, Suprema Corte de Justicia de la Nación, Tribunal Electoral del Poder Judicial de la Federación, 2010, pp. 3 e ss. 
mente elevato di riforme, talune estrinsecatesi anche nella forma di revisioni totali. ${ }^{2}$

La prima Costituzione fu approvata il 23 ottobre 1857 dal Congreso del Estado, per poi essere promulgata sei giorni dopo dal gobernador constitucional. Il testo, che si segnalava, tra l'altro, per il riconoscimento della religione cattolica come religione di Stato, ${ }^{3}$ restò in vigore per un periodo piuttosto limitato. Già nel 1861, infatti, una serie di riforme vennero a stravolgerne i contenuti, al punto che si è soliti indicare il risultato delle revisioni come una nuova Costituzione. Una Costituzione di cui una parte della dottrina storicistica ha tra l'altro contestato la validità, sull'assunto che avrebbe fatto difetto una formale approvazione del testo da parte del Congresso. ${ }^{4}$ Sul punto, l'opinione maggioritaria è, tuttavia, in senso decisamente opposto, ${ }^{5}$ per cui pare che possa confermarsi la validità a tutti gli effetti del testo adottato nel 1861. Un testo che, pur avendo anch'esso avuto una vita piuttosto breve, ha segnato una tappa importante nella storia costituzionale dello Stato (ma probabilmente del Messico nel suo complesso), poiché ha posto il principio dell'elezione diretta del vertice del potere esecutivo, ${ }^{6}$ che sino ad allora veniva designato, tanto a livello federale quanto nei singoli stati, attraverso un'elezione indiretta. Sul piano della garanzia dei diritti, poi, è da rilevare che la Costituzione del 1861 si segnalava per l'incorporazione di un catalogo piuttosto articolato, nel quale spiccava, tra le altre, la tutela della libertà di culto.

Le novità introdotte nel 1861 sono state mantenute, con qualche ritocco (come, ad esempio, l'introduzione del divieto di rielezione del governatore), nella Costituzione del 1868, la prima Costituzione dello stato connotata da una certa longevità. $\mathrm{Fu}$, infatti, solo dopo la fine della rivoluzione, ed in concomitanza con l'adozione di una nuova Costituzione a livello federale, che anche lo stato di Aguascalientes si dotò di un nuovo testo

2 Una raccolta completa dei testi costituzionali statali è riportata in idem, pp. 77 e ss.

3 L'art. 5o. così recitava: "La Religión del Estado de Aguascalientes es la Católica, Apostólica, Romana".

4 La tesi è sostenuta da Jesús Gómez Serrano, in Santos Olivo de los, Isidro, Aguascalientes. Historia de las instituciones jurídicas, México, Senado de la RepúblicaUNAM, Instituto de Investigaciones Jurídicas, 2010, p. 115.

5 Cfr., in part., de la Torre Rangel, Jesús Antonio, "El liberalismo jurídico de los rojos de Aguascalientes", Revista de Investigaciones Jurídicas, n. 31, 2007, p. 864.

6 L'art. 81 della Costituzione stabiliva che "[1]a elección de gobernador propietario y suplente será popular directa en los términos que fije la ley". 
fondamentale, approvato dal Congresso statale il 3 settembre e pubblicato sei giorni dopo.

Da notare, peraltro, è che, in relazione alla qualificazione dell'esercizio del potere che è alla base della Costituzione del 1917, il piano formale e quello sostanziale divergono in misura significativa. Per un verso, nell'epigrafe si esplicita l'esercizio del potere di revisione della Costituzione del 18 ottobre 1868, con il che parrebbe di potersi parlare di una continuità da un punto di vista ordinamentale. Per altro verso, sul piano sostanziale, è altrettanto evidente che si tratti di una riscrittura compiuta, caratterizzata altresì da profonde modifiche contenutistiche, legate ai cambiamenti conseguenti alla rivoluzione.

Se, dunque, si adotta — come pare più corretto - un criterio di valutazione di tipo sostanziale, ben può dirsi che nel 1917 sia stato esercitato un potere costituente in senso proprio, e non soltanto un potere costituente che, mutuando un'espressione non in uso in Italia, ma diffusa nel mondo ispanofono (oltre che in quello francofono), può definirsi "derivato".

Questa impostazione incontra, tuttavia, qualche difficoltà allorché si vada oltre l'orizzonte temporale del 1917. La Costituzione, infatti, ha conosciuto profondi cambiamenti, segnatamente nel 1950, quando si è fatto luogo ad una revisione estremamente lata del testo, al punto che quella che era la struttura originaria ne è risultata stravolta. Solo per dare una misura dell'impatto delle modifiche, può porsi mente al fatto che, nella versione originaria, la Costituzione constava di soli 73 articoli, mentre oggi gli articoli arrivano al numero 95, ma sono molti di più, essendo stati introdotti anche articoli contrassegnati, oltre che dal numero, anche da lettere. Se, dunque, si seguisse in maniera rigorosa — come si è sin qui suggeritoun'impostazione sostanziale, allora la Costituzione vigente di Aguascalientes dovrebbe probabilmente essere datata 1950.

L'alternativa tra questa soluzione e quella strettamente formale, che vorrebbe la Costituzione riferita addirittura al 1868, porta ad esiti comunque insoddisfacenti, perché, nonostante le difficoltà tecniche che possono rintracciarsi, e di cui si è dato conto, la storia costituzionale messicana porta a mettere in risalto la rottura ordinamentale prodottasi, a livello generale, nel 1917; di tale rottura è espressione anche la Costituzione statale di Aguascalientes, al pari della Costituzione federale e di quelle di molti altri stati che compongono la Federazione. È, in definitiva, un criterio eminentemente storico a dover guidare nella datazione: il dato giuridico 
mostra, infatti, una commistione tra rottura e continuità tale da non poter essere sciolta, se non a prezzo di una qualche forzatura che rischia di dar luogo a scelte che si rivelino arbitrarie.

\section{LA “MANUTENZIONE COSTITUZIONALE”}

Una seconda osservazione su cui conviene brevemente soffermarsi attiene alla prassi delle riforme costituzionali. Scorrendo il testo costituzionale si incontra un elevatissimo numero di articoli e di disposizioni che sono stati modificati nel corso degli anni, anche molto recentemente. Ora, questo numero di riforme costituzionali può prestarsi a due differenti letture.

Da un primo punto di vista, si può apprezzare l'attentissima "manutenzione costituzionale" che le autorità dello stato di Aguascalientes hanno posto in essere in maniera costante nel corso del tempo.

Per riprendere le parole di Isidro de los Santos Olivo, "[1]a técnica de la reforma constitucional implica - contrariamente a lo que se pueda pensar y a lo que se ha vivido en la accidentada vida constitucional del estado y del país - el primer mecanismo de defensa de la Constitución". ${ }^{8}$ Questo, come è chiaro, se si ha come presupposto che "la modificación a la Constitución resulta políticamente conveniente, cuando es jurídicamente necesaria". 9

In effetti, la Costituzione di Aguascalientes si dimostra molto attenta all'evoluzione sociale ed all'emergere di nuove esigenze di protezione. Tra i molteplici riferimenti che potrebbero farsi a titolo esemplificativo, può evocarsi la riforma del 2014 relativa all'art. 7, in materia di diritto degli individui ad un ambiente salubre, ${ }^{10}$ con cui si è costituzionalizzato un diritto che in maniera sempre più nitida è andato emergendo nel panorama del costituzionalismo mondiale. Ancor più significativo, da questo punto

7 L'espressione richiama quella utilizzata, in riferimento alla legislazione, da Alessandro Pizzorusso: cfr. Pizzorusso, Alessandro, La manutenzione del libro delle leggi ed altri studi sulla legislazione, Torino, Giappichelli, 1999.

8 Cfr. Santos Olivo, Isidro de los, op. cit., p. 129.

9 Ibidem.

10 Art. 7: "Toda persona tiene derecho a un medio ambiente sano para su desarrollo y bienestar, así como el deber de protegerlo y conservarlo. Todas las autoridades en la esfera de sus atribuciones velarán por la conservación y fomento de los recursos naturales del estado". 
di vista, è l'inserimento, nel 2016, di un ultimo comma all'art. 6, che ha sancito il diritto ad accedere liberamente ad Internet. ${ }^{11}$

Quest'ultima revisione, agli occhi di un giurista italiano, è particolarmente significativa, perché implica una scelta di campo ben precisa, sulla quale, in Italia, ormai da anni ci si interroga. Che Internet abbia assunto una importanza assolutamente centrale nella vita della gran parte dei consociati è un dato che non richiede particolari commenti. Che questa importanza si traduca nella necessità di inserire Internet all'interno della Costituzione è, invece, una questione aperta: da un lato, si pongono coloro secondo i quali sostengono che il costituzionalizzare il diritto di accedere ad Internet (proprio nella forma per cui si è optato nello stato di Aguascalientes) avrebbe l'effetto di rafforzare le tutele offerte agli individui; dall'altro lato, si pongono, invece, coloro che ritengono non necessario questo inserimento, in quanto la garanzia dell'accesso ad Internet sarebbe comunque rintracciabile in via implicita nel testo costituzionale.

Al momento, in Italia è questa seconda opzione che ha prevalso: sarebbe quindi interessante poter fare, tra qualche anno, una comparazione relativa agli effetti prodotti dall'inserimento della garanzia dell'accesso ad Internet nella Costituzione dello Stato di Aguascalientes ed all'impatto della mancata esplicitazione di questo diritto nella Costituzione italiana. In particolare, sarebbe da capire quanto la previsione costituzionale di un obbligo per i pubblici poteri di garantire l'esercizio del diritto di accesso possa realmente incidere sull'attività che venga posta in essere e sulla sua efficacia.

La manutenzione costituzionale non copre, peraltro, solo l'evoluzione sociale, ma tende anzi ad assecondare mutamenti significativi del quadro normativo generale. Emblematico è, al riguardo, il testo dell'art. 2 della Costituzione, come modificato nel 2015, che rispecchia in modo pressoché totale la riforma della Costituzione federale in materia di protezione dei diritti fondamentali. ${ }^{12}$

\footnotetext{
11 L'ultimo comma dell'art. 6 così recita: “El estado y los municipios garantizarán el derecho de acceso libre a Internet, para tal efecto, establecerán los mecanismos y políticas públicas necesarias para asegurar progresiva y de manera gradual la efectividad de este derecho".

12 L'art. 2 è così formulato: "En el estado de Aguascalientes, todas las personas gozarán de los derechos humanos reconocidos en la Constitución Política de los Estados Unidos Mexicanos, en el derecho internacional en materia de derechos humanos, en las leyes generales expedidas por el Congreso de la Unión, la presente Constitución y en Esta obra está bajo una Licencia Creative Commons Atribución-NoComercial-SinDerivar 4.0 Internacional, IIJ-UNAM.
} 
Adottando un diverso punto di vista, e sempre riprendendo quanto affermato da Isidro de los Santos Olivo, se si guarda alla storia della Costituzione di Aguascalientes:

...se puede apreciar que algunos principios establecidos por la teoría de la Constitución no se han afianzado en la cultura político constitucional del estado. Es el caso de los principios de rigidez y el de supremacía constitucional. La Constitución es reformada sin ningún tipo de límites y se convierte, en la materialidad y de manera paradójica, en la norma más alterada del ordenamiento jurídico estatal. ${ }^{13}$

La contrapposizione tra questa lettura e quella che si è in precedenza proposta fa emergere una divaricazione nel concepire la Costituzione che può prestarsi ad un esame in prospettiva comparatistica. Un esame che si rivela di grande interesse, sebbene non possa condurre ad individuare quale sia la nozione più efficace per dare stabilità al sistema, per il semplice fatto che la funzionalità e l'efficacia di un sistema possono essere assicurate nei modi più vari, ed in questi modi possono rientrare tanto una intensa attività di adeguamento del testo costituzionale quanto una sua stabilizzazione così marcata da evocare il concetto di cristallizzazione. Due esempi, tra $\mathrm{i}$ molti che potrebbero proporsi, possono risultare, nella loro contrapposizione, adeguatamente esplicativi.

Da un lato si pongono gli Stati Uniti, la cui Costituzione federale, dal 1787, ha subito soltanto ventisette emendamenti, dei quali tra l'altro i

las leyes locales, así como de las garantías para su protección, cuyo ejercicio no podrá restringirse ni suspenderse, salvo en los casos y bajo las condiciones que la propia Constitución federal establece.

Las normas relativas a los derechos humanos se interpretarán siempre de conformidad con la Constitución Política de los Estados Unidos Mexicanos, con los tratados internacionales de la materia y con la presente Constitución, favoreciendo en todo tiempo a las personas la protección más amplia.

Todas las autoridades, en el ámbito de sus competencias, tienen la obligación de promover, respetar, proteger y garantizar los derechos humanos de conformidad con los principios de universalidad, interdependencia, indivisibilidad y progresividad. En consecuencia, el Estado deberá prevenir, investigar, sancionar y reparar las violaciones a los derechos humanos, en los términos que establezca la ley".

Dalla lettura dell'articolo, si evince la quasi compiuta sovrapponibilità del testo rispetto a quello dei primi tre commi dell'art. 1 della Costituzione federale, introdotti con la revisione costituzionale del 2011.

13 Santos Olivo, Isidro de los, op. cit., p. 130. 
primi dieci pressoché contestuali all'approvazione dell'articolato costituzionale. La quasi irrilevanza dell'attività di manutenzione costituzionale non ha, ovviamente, impedito l'evoluzione del sistema, che anzi ha conosciuto, come noto, cambiamenti quanto mai profondi. La ritrosia a porre mano a riforme costituzionali si è legata, tuttavia, all'esigenza di preservare l'autorità della Carta fondamentale. ${ }^{14}$ In questa preservazione risiede proprio uno dei fondamenti della stabilità del sistema istituzionale, che si è associata ad una evoluzione che ha percorso i canali dell'interpretazione e dell'attuazione legislativa.

Sul versante opposto si colloca l'esperienza francese della Quinta Repubblica, la cui Costituzione, per il primo decennio di vigenza, è stata ritenuta alla stregua di "un vestito cucito addotto al Generale de Gaulle", ma che poi è andata progressivamente accreditandosi come un testo in cui tutti potessero riconoscersi. ${ }^{15}$ Ebbene, questo processo è stato certamente agevolato da alcune importanti riforme costituzionali, che negli ultimi venticinque anni hanno assunto una funzione di consolidamento del sistema, attraverso una accelerazione significativa della frequenza con cui si ricorre ad interventi di manutenzione costituzionale.

Il confronto tra Stati Uniti e Francia, nella misura in cui dimostra come non esista un modello astratto che sia idoneo ad assicurare la piena esplicazione della supremazia costituzionale, lascia in sospeso la valutazione della prassi instauratasi nello stato di Aguascalientes. Una prassi che, in ogni caso, non pare che possa essere bollata come patologica.

Anzi, pare di poter dire che le modifiche frequenti, se ponderate, risultano, comunque, idonee a garantire una corrispondenza tra le esigenze del sistema e l'evoluzione della Costituzione. Questo, almeno, è quello che suggerisce l'accostamento con l'esperienza italiana, nella quale la mancata manutenzione costituzionale è stata additata, da una parte della

14 Questa posizione venne espressa con forza già da James Madison nei Federalist Papers, n. 49 (5 febbraio 1788), www.foundingfathers.info/federalistpapers/fedindex.htm.

15 Al punto che François Mitterrand, uno dei più accesi contestatori, negli anni sessanta, del sistema (da lui stesso identificato in un "colpo di Stato permanente": $c f r$. Mitterrand, François, Le coup d'État permanent, Paris, Plon, 1964), una ventina di anni dopo, nel 1986, da presidente della Repubblica, non ebbe esitazione ad individuare nella Costituzione ( LLa Constitution, toute la Constitution, rien que la Constitution») la stella polare capace di guidare il sistema nel periodo, fino ad allora inedito, della coabitazione tra una maggioranza parlamentare (ed un governo) espressione di un indirizzo politico opposto rispetto a quello del capo dello Stato (la c.d. cohabitation). 
dottrina, come la causa essenziale dell' "invecchiamento" della Costituzione repubblicana, in vigore dal 1948. Sul punto, peraltro, il discorso si fa complesso, poiché se è vero che taluni aggiornamenti sarebbero stati (e sarebbero) necessari, è anche vero che il processo di invecchiamento si è associato ad uno strisciante affievolimento dell'autorevolezza della carta, che è stato dovuto, probabilmente, non tanto alla sua mancata riforma in sé e per sé, quanto semmai alla frequente invocazione di riforme che, giunte ad uno stadio più o meno avanzato di elaborazione, non sono state alla fine adottate, con il risultato di mantenere in vita quelle disposizioni che, proprio perché oggetto di potenziale cambiamento, erano state criticate anche molto diffusamente. Ed il succedersi di questi tentativi abortiti (sovente anche perché molto - troppo- ambiziosi nel ridisegnare un testo che, complessivamente, continua a dar prova di efficacia) ${ }^{16}$ ha portato, nel tempo, ad una radicalizzazione del dibattito sulla necessità e sulla opportunità di operare riforme costituzionali, che sta producendo una sorta di stallo le cui conseguenze sono, in massima parte, misurabili in termini di impatto negativo sull'autorevolezza della Costituzione vigente.

\section{IL PARADOSSO DELLE RIFORME ${ }^{17}$}

Il terzo elemento su cui è il caso di porre, per quanto in maniera sommaria, l'accento riguarda il collegamento tra la manutenzione costituzionale ed il procedimento di riforma costituzionale.

Per riformare la Costituzione statale di Aguascalientes si richiede una maggioranza molto elevata (i due terzi dell'assemblea legislativa), oltre

16 L'ultimo tentativo in ordine di tempo è stato quello della riforma sottoposta a referendum costituzionale il 4 dicembre 2016, referendum che ha visto una maggioranza dei voti contrari alla revisione. Su tale riforma e sugli esiti della sua mancata approvazione, sia consentito rinviare a Passaglia, Paolo (ed.), The 2016 Italian Constitutional Referendum: Origins, Stakes, Outcome, The Italian Law Journal, Special Issue, 2017, disponibile alla pagina www.theitalianlawjournal.it/specialissues/(data di ultimo accesso: 12 agosto 2018).

17 Il titolo del paragrafo evoca quello del contributo di Zagrebelsky, Gustavo, "I paradossi della riforma costituzionale", Politica del diritto, 1986, pp. 165 e ss., in cui però il concetto di paradosso viene associato alle riforme in un'accezione completamente diversa, ad evocare cioè l'estrema difficoltà di modificare il sistema istituzionale in conseguenza dell'incapacità decisionale del sistema stesso, a testimonianza quindi della necessità che le riforme vengano fatte. 
alla metà delle assemblee municipali. ${ }^{18} \mathrm{~A}$ ben vedere, però, queste soglie elevate non si dimostrano particolarmente difficili da raggiungere, in ragione del numero ridotto di deputati: per coagulare i voti dei due terzi dei deputati, un testo deve essere approvato da diciotto deputati su ventisette. Con numeri così bassi, tenendo anche conto del sistema elettorale che, come noto, tende a premiare la formazione politica maggioritaria, non appare così difficile trovare un accordo che sia sufficiente. Ad esempio, attualmente il partito di maggioranza ha tredici deputati: perché i due terzi siano raggiunti, basta che riesca ad avere altri cinque voti.

Si spiega, forse, proprio con il ridotto numero di deputati l'apparente paradosso per cui un procedimento di riforma costituzionale che richiede una soglia molto elevata convive con una frequenza notevole di leggi di riforma costituzionale.

Ci si può interrogare se non fosse il caso di introdurre ulteriori aggravamenti al procedimento di revisione, onde garantire il sistema contro eventuali riforme costituzionali troppo legate all'indirizzo politico contingente. Elevare oltre i due terzi il quorum approvativo della revisione parrebbe, tuttavia, eccessivo, poiché si rischierebbe di scivolare verso la richiesta di una unanimità di fatto. Una forma alternativa sarebbe quella di prevedere la possibilità di ricorrere ad un referendum, come strumento oppositivo rispetto alla decisione della maggioranza: la minoranza assembleare o un certo numero di cittadini o di municipalità potrebbero essere legittimati a richiedere lo svolgimento del referendum, in maniera analoga a quanto viene previsto nell'art. 138 della Costituzione italiana, salvo che, nel caso di Aguascalientes, al referendum dovrebbe potersi far luogo a prescindere dalla maggioranza raggiunta in sede assembleare, proprio per la facilità di raggiungere la soglia dei due terzi. ${ }^{19}$

18 L'art. 94 della Costituzione così dispone: "La presente Constitución puede ser adicionada o reformada con los requisitos siguientes:

I.- Iniciada la reforma y aprobada por los votos de las dos terceras partes del número total de diputados, se pasará a los ayuntamientos con los debates que hubiere provocado, para su discusión; si la mayoría de los ayuntamientos aprobaren la reforma o adición, ésta será declarada parte de la Constitución;

II.- Si transcurrieren quince días desde la fecha en que los ayuntamientos hayan recibido el proyecto de reforma, sin que se hubiere recibido en el Congreso el resultado de la votación, se entenderá que aceptan la reforma o adición".

19 Nella Costituzione italiana, il referendum può essere richiesto, invece, soltanto se il Parlamento non abbia approvato il testo con una maggioranza dei due terzi: l'idea è infatti quella che, con un quorum così elevato, sussista un consenso generalizzato nei con- 


\section{UNA COSTITUZIONE "RICCA"}

A mo' di conclusione, pare opportuno spendere qualche parola sui contenuti concretamente presenti nella Costituzione dello Stato di Aguascalientes.

Pur senza coltivare l'ambizione di analizzare specifici istituti, è comunque possibile, anche di primo acchito, mettere in risalto la ricchezza di contenuti che connota la Costituzione. Una ricchezza che si apprezza sia sotto il profilo della quantità di materie trattate sia sotto il profilo del grado di precisione di molte disposizioni.

Per quel che riguarda le materie inserite all'interno della Costituzione, si può constatare come essa estenda la sua normazione su un vasto novero di materie, onde coprire, sostanzialmente, l'integralità della c.d. "materia costituzionale".

Va peraltro detto che, rispetto a gran parte delle Costituzioni statali messicane, quella di Aguascalientes è relativamente breve, nel senso che il numero di articoli è piuttosto contenuto ed anche la lunghezza del testo è, nel complesso, assai minore dell'id quod plerumque accidit. Ciò posto, è comunque indubbio che si tratti di una Costituzione che può definirsi "lunga” (in senso tecnico), perché tratta, oltre che dell'organizzazione dei poteri, anche dei diritti individuali.

A ben vedere, anzi, c'è molto di più di quanto ci si possa aspettare. Due esempi possono, al riguardo, prospettarsi.

Il primo riguarda il catalogo dei diritti fondamentali, che è molto articolato e che copre sia i diritti di libertà sia - soprattutto - i diritti sociali. L'elenco dei diritti parte, all'art. 4, con il riconoscimento della centralità della famiglia come cellula sociale fondamentale, per poi enfatizzare la necessità di approntare una tutela compiuta nei confronti nei minori, sotto il profilo dell'intervento pubblico, ma anche nel quadro della tutela dell'identità personale sin dalla nascita. Sempre nel quadro familiare, si afferma il principio di eguaglianza tra i sessi, con una formulazione che, peraltro,

fronti della riforma. Il punto fondamentale, al riguardo, risiede, ovviamente, nei numeri: a fronte dei 27 deputati di Aguascalientes, la Camera dei deputati italiana è composta da 630 membri, mentre il Senato della Repubblica da 315 membri elettivi oltre ai senatori di diritto (fino a 5) ed a vita (gli ex-presidenti della Repubblica); numeri così ampi permettono di ritenere che, quando nelle assemblee si abbia un amplissimo consenso, ciò sia indice di un ampio consenso anche a livello sociale. 
trascende i semplici legami familiari. All'art. 4 si afferma in maniera molto articolata anche il diritto alla cultura ed il diritto alla salute. All'art. 5, ad essere protetta è la proprietà privata, di cui si mette in evidenza, tra l'altro, la necessaria funzione sociale. L'art. 6o. è invece dedicato al diritto all'istruzione: significativamente, il diritto di accesso ad Internet è stato inserito al termine di una serie molto analitica di disposizioni che mirano a rendere effettivo ed efficace il diritto alla formazione. L'elenco si chiude, "provvisoriamente", con le previsioni in materia di ambiente (art. 7) e di sviluppo sostenibile (art. 7A), quest'ultimo disegnato attraverso azioni dei pubblici poteri nelle quali gli obiettivi di stampo prettamente economico si compenetrino con l'attenzione rivolta al benessere della collettività.

A completamento di questo elenco, si pone poi il capitolo "Del sistema de justicia penal acusatorio, el Ministerio Público, el sistema de seguridad pública y los derechos humanos". Gli articoli 58A e 58B fissano le linee essenziali del processo penale di stampo accusatorio, l'art. 58C si concentra essenzialmente sui rapporti tra pubblici ministeri e giudici, mentre l'art. 58D attiene alla fase decisoria del processo; alla responsabilità penale dei minori è dedicato l'art. 58E. Gli articoli 59 e 60 riguardano la figura del pubblico ministero, l'art. 61 si concentra invece sulle forze di polizia.

A garanzia del rispetto dei diritti fondamentali, precipuamente nell'ambito del procedimento penale, è istituita la Comisión Estatal de Derechos Humanos (art. 62). Infine, con una previsione che esorbita rispetto al processo penale, si stabilisce, in chiusura del capitolo, all'art. 62A, il diritto di accesso alle informazioni che riguardino l'individuo.

La marcata attenzione al procedimento penale ed alla tutela dei diritti in esso coinvolti è significativo che abbia uno spazio così ampio nella Costituzione statale, giacché dimostra l'estrema delicatezza di questa materia e la preoccupazione che il Costituente ha avuto nel proteggere il più possibile l'individuo quando si trovi di fronte alla potestà punitiva dello Stato. Forse l'attenzione al collegamento tra diritti e procedimento penale può essere stata ispirata dall'osservazione dell'ordinamento degli Stati Uniti, e della giurisprudenza della Corte Suprema federale, che si è grandemente sviluppata proprio su queste materie.

Il secondo ambito al quale conviene accennare, per il suo valore esemplificativo, è quello della disciplina del sistema elettorale per il Congreso del Estado. A differenza di quanto generalmente si constata nelle costituzioni europee, la Costituzione dello Stato di Aguascalientes detta regole 
precise, a testimonianza di quanto sia fondamentale la legge elettorale per il funzionamento delle istituzioni statali. La materia elettorale è un buon esempio anche del livello di precisione delle disposizioni. Leggendo la Costituzione, infatti, si ha un quadro molto dettagliato di come il Congreso del Estado viene composto.

Ora, guardando il testo con gli occhi di un giurista europeo, può apparire eccessivo dedicare tanto spazio e diffondersi in discipline tanto dettagliate: le Costituzioni europee, infatti, generalmente non trattano del sistema elettorale se non in modo molto limitato, lasciando alla legge il compito di concretizzare anche le scelte più rilevanti. A ben vedere, peraltro, la scelta di integrare la regolamentazione del sistema elettorale all'interno della Costituzione non può essere semplicemente bollata come un eccessivo irrigidimento. Lasciare alla legge ordinaria il compito di disegnare il sistema elettorale può ingenerare, infatti, difficoltà assai rilevanti, segnatamente per l'impatto che le modifiche delle modalità di selezione della rappresentanza possono avere sul funzionamento del sistema nel suo complesso. L'esperienza italiana, da questo punto di vista, è emblematica: la Costituzione repubblicana, sebbene non abbia esplicitato l'opzione in favore del proporzionale, lo ha chiaramente presupposto, con il risultato che, allorché si passò, all'inizio degli anni novanta, ad un sistema prevalentemente maggioritario, ci si accorse che le garanzie approntate dalla Costituzione non erano sufficientemente efficaci una volta traslate in un sistema in cui una parte politica avesse la possibilità di soverchiare l'altra.

Probabilmente, se si fosse scelta la via di costituzionalizzare il sistema elettorale, come avvenuto - tra l'altro - nell'esperienza dello stato di Aguascalientes, ciò avrebbe reso più difficili i cambiamenti di sistema elettorale che hanno caratterizzato gli ultimi lustri e, al contempo, avrebbe assicurato uno sviluppo più armonico al sistema istituzionale. Benefici di questo genere, in effetti, sembrano lungi dal potersi trascurare, al punto che l'osservazione di esempi stranieri, come quello qui in esame, potrebbero suggerire qualche proficuo ripensamento. 